\title{
User interface metaphor in virtual reality using VRML
}

\author{
DAVID R. NADEAU \\ San Diego Supercomputer Center, San Diego, Califormia
}

\begin{abstract}
A metaphor is the description of an unfamiliar thing in terms of a familiar one. A user interface metaphor is a computer user interface design that presents computer abilities in terms of familiar real-world abilities. Such a metaphoric design enables a user to use existing knowledge about the real world when interacting within the computer's world. Common user interface metaphors include the desktop metaphor of personal computers and the document metaphor of the Web. In both metaphors, computer information objects are presented as files, folders, and pages of text. The recent development of the virtual reality modeling language (VRML) enables the design of new, inherently 3-D user interface metaphors for the Web. This paper introduces the essential features of VRML and discusses issues in the design of 3-D user interface metaphors using VRML.
\end{abstract}

As the World-Wide Web has evolved, it has grown to embrace many media types, from text to images, sounds, movies, and now 3-D virtual environments using the virtual reality modeling language (VRML; see Ames, Nadeau, \& Moreland, 1996; Bell, Parisi, \& Pesce, 1995). VRML files contain platform-neutral ASCII text instructions that describe the size, position, color, texture, and lighting of 3-D shapes and scenery. When displayed by a VRML browser application, the user can interactively walk about and examine scene content from arbitrary viewpoints. Similar to the anchor text of the hypertext markup language (HTML; see Raggett, 1995), VRML worlds may contain anchor shapes that, when clicked upon, upload a new VRML scene from the Web and into the browser.

To appreciate the problems and potential of the future 3-D Web, it is important to understand the underlying technology that will create it and some of the user interface issues that will guide its designers. The sections that follow provide a brief introduction to VRML and then discussion of user interface metaphors for virtual environments.

\section{INTRODUCING VRML}

\section{The Style of VRML}

VRML's language style mimics that of English-language instructions you might use to direct a colleague to draw something. Imagine, for instance, that you want to instruct your colleague to draw two cubes side by side. The

This paper is a result of software development and user interface work done in collaboration with John L. Moreland of the San Diego Supercomputer Center and Hans Sieburg of the University of California San Diego Psychiatry and Math departments. Correspondence should be addressed to D. R. Nadeau, San Diego Computer Center, P.O. Box 85608, San Diego, CA $92186-9784$ (e-mail: nadeau@sdsc.edu). first cube is to be drawn in green ink, and the second is to be drawn in blue. Here is such an instruction list:

1. Pick up a green pen.

2. Draw a cube.

3. Pick up a blue pen.

4. Move to the right of the green cube.

5. Draw a cube.

This list has two types of instructions: property-change instructions $(1,3$, and 4$)$, and shape-drawing instructions ( 2 and 5). Property-change instructions change the way in which future shapes will be drawn by specifying what color to use, where to draw, and so on. Shape-drawing instructions use the current set of properties, such as the pen color, and direct the creation of marks on your colleague's paper.

VRML uses a similar instruction style to direct the activities of a virtual pen drawing in a 3-D space. VRML instructions, called nodes, change pen properties or draw shapes. For example, the following VRML instructions draw two cubes, one in green and the other in blue:

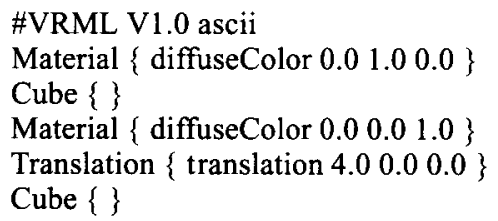

The first line in the VRML file identifies the data as conforming to VRML's Version 1.0 language specification. Future versions of the VRML language specification will use their own identification lines. The second line sets the VRML virtual pen's ink color by using a "material" node. VRML colors are specified as a mixture of varying amounts of red, green, and blue-like mixing paints on a palette. In the example above, the pen color "0.0 1.0 0.0 " indicates that no red, lots of green, and no blue are to be mixed together to create a pure 
green ink. The third line directs the VRML virtual pen to draw a "cube." Since the pen color has just been set to green, the cube is drawn in green. The fourth line changes the virtual pen color again. The color mix specifies that no red, no green, and lots of blue are to be mixed together to create a pure blue ink. The fifth line directs the pen to move, or "translate," to a new location in the 3-D drawing space. Using three distance values, the author directs pen motion left to right, bottom to top, and back to front. In the above example, the pen is moved " 4.0 " units to the right, " 0.0 " units up, and " 0.0 " units forward. This places the pen to the right of the previously drawn green cube. Finally, the sixth line directs the pen to draw a second "cube." Since the pen color is now blue, the new cube is drawn in blue.

The above example used only three types of VRML nodes: Material, Translation, and Cube. There are several dozen additional node types that provide drawing instructions for changing lighting and shading properties, drawing complex faceted shapes, and grouping together sets of instructions in to user-defined hierarchies. By creating sequences of these VRML drawing instructions, authors can create highly detailed, realistic 3-D virtual environments on the Web.

\section{Anchors in VRML}

Perhaps the most important feature of VRML is its ability to define 3-D "anchor shapes." Like the underlined text anchors in HTML pages, VRML anchor shapes are objects in the virtual world that, when clicked upon, jump the user to another VRML world on the Web.

To define an anchor shape, the VRML author groups together any list of VRML nodes and provides an anchor URL for the destination VRML file. Such a group of VRML nodes can draw anything as the anchor shape. The author can, for instance, create anchor shapes out of doors in a room, paintings on a wall, books on a shelf, or drawers in a desk. Anchor shapes can be arbitrarily large and complex shapes, such as a mountain peak on a 3-D relief map or a planet in a virtual solar system. The choice is up to the VRML author.

\section{THE ARCHITECTURE METAPHOR}

\section{User Interface Metaphors}

A user interface metaphor applies users' existing knowledge about how the real world works to give them a head start in understanding how a fictional computer world works. Perhaps the most common presentation metaphor in use today is that of the desktop metaphor.

Developed by Xerox PARC and made popular by Apple's Macintosh, the desktop metaphor presents computer information and operations in familiar real-world terms: that of documents sitting on a desk. Like realworld documents, desktop metaphor documents can be moved about, slipped into folders, and thrown out by dropping them in the trash. Screen windows simulate a stack of documents cluttering up a desk.
Using any metaphor, users need to create, destroy, find, view, edit, organize, and transport information. These operations are grouped into two categories: interaction operations, and navigation operations. Create, delete, view, and edit are interaction operations and typically require application-specific tools, such as word processors, drawing programs, spreadsheets, and databases.

The desktop metaphor itself is concerned only with navigation operations to find, organize, and transport information. These navigational operations are the principle interest when considering the design of a metaphor appropriate for 3-D virtual environments on the Web.

\section{Architecture Metaphors}

An architecture metaphor for information presentation constructs a 3-D structure in which information resides. The metaphor may, for instance, construct rooms in a building, buildings in a city, cities in a country, and so forth. Navigating the information space of an architecture metaphor invokes a user's existing familiarity with navigating through cities and buildings in the real world. Landmarks in the virtual space help to direct the user to interesting places and give the user visual cues to help remember where important information is kept.

Information in such an architectural metaphor can include a variety of media types. For instance, 3-D shapes can line the shelves of a warehouse, while 2-D images hang on the walls of a virtual gallery, and movie reels and books occupy shelves in a metaphoric film vault or library.

By navigating the architectural space, users can find, organize, and transport information, structuring their virtual environment in ways similar to those used in the real world. Like the real world, the organization strategies employed by users are up to them. Rooms, shelves, and closets can be as organized, or chaotic, as the user desires.

\section{What Is Essential and Appropriate?}

The traditional role of virtual reality has been to construct compellingly realistic environments. In an aircraft simulator environment, for instance, it is essential that the pilots suspend disbelief and accept the reality of the virtual environment so that their actions and reactions ring true. For instance, pilots must not want to crash. Their pulses must pound when unexpected virtual turbulence shakes them or when fog socks in a virtual airport. Detail in such an environment is important in order to enhance the realism and guarantee suspended disbelief.

When creating a user interface metaphor, the designer must examine closely the metaphor's real-world subject and mimic only those features essential for the metaphor. Detail is not as important. For example, a user's real-world desktop may have Coke cans, coffee mugs, crumpled candy wrappers, assorted paper weights, stray pens, and a tangle of paper clips. In an entertaining simulation, a virtual desktop could include all of this clutter. In a user interface metaphor, none of these are necessary. Instead, 
the traditional desktop metaphor focuses upon only the essential and appropriate components of a desktop: documents, folders, and a nearby trash can.

When designing a 3-D user interface metaphor, the real world is examined with this same eye toward what is truly essential versus what is fun in a realistic simulation. This is a departure from current virtual reality work that focuses largely on making worlds as realistic and detailed as possible.

To begin, rooms and walls are essential to an architecture metaphor. These serve to establish a sense of space and enable the user to structure and organize information. Doors between the rooms provide a convenient and familiar connection mechanism. These too are essential to the metaphor. What about doorknobs? The presence of a doorknob in the real world is a clue that a door is functional and meant to be opened. A case could be made that doorknobs are essential to the architecture metaphor. Do metaphorical doorknobs need to turn? Are latch mechanisms necessary? Do metaphorical doors need weather stripping? Screen doors? Welcome mats?

The temptation always is to add detail, because it is fun. However, each bit of additional detail can distract from the core function of a metaphor feature. An excessively ornate door may look outstanding but leave the doorknob hard to find.

As another example, what types of rooms are necessary in a virtual building? Libraries, offices, storerooms, and closets seem to make sense. Does a virtual environment need restrooms and a kitchen? It seems unlikely. Similarly, at the city level, what is essential and appropriate? Public libraries, office buildings, recreation areas, meeting spaces, and stores each seem appropriate. What about restaurants, dry cleaners, and gas stations? Are cars necessary? Roads? Parking meters? Traffic jams?

This critical analysis of the real world is necessary when picking and choosing aspects to mimic in a virtual world metaphor. It is tempting to mimic the real world closely, creating realistic and entertaining virtual environments. In so doing, the author brings along features that may not be necessary in a virtual world, such as parking lots and traffic jams. On the other hand, if too few realworld features are included, the metaphor's result can be so different from the familiar real world that users are unable to utilize their real-world knowledge to aid them in navigating the virtual world. Gravity, for instance, may be an essential feature for virtual worlds, though getting hurt when you fall is a feature perhaps best left out.

The ease with which alternate realities can be constructed using VRML enables the development of a wide variety of new user interface metaphors. Each new VRML file on the Web can implement a different metaphor, mimic different sets of real-world features, or even depart entirely from real-world mimicry. Instead of the well-ordered user interface metaphors of today's computers, the future Web is likely to be a fascinating, but chaotic, environment of clashing metaphors.

\section{METAPHOR BREAKDOWN}

Once a metaphor is established, such as the architecture metaphor, where does it break down? What features of the underlying technology will make consistent application of the metaphor problematic?

\section{Connectivity}

In the real world, if Room A has a door to Room B, then Room B must have a door back to Room A. It is, in fact, the same door. We could state this as a connectivity law:

If $\mathrm{A}$ connects to $\mathrm{B}$, then $\mathrm{B}$ must connect to $\mathrm{A}$.

In VRML, this is not necessarily true. A VRML file describes the shapes, and anchors, visible from that world. Room A's VRML file describes A's room, and a door anchor shape that, when clicked upon, jumps the user to Room B. Room B's VRML file describes B's room, independent of Room A's description. It may have been created before Room A was built and need not contain a door back to $A$. When linked together, the user can jump through A's door to Room B but can never get back again. The metaphor has broken down.

\section{Single Existence}

In the real world, there can be at most a single instance of any object or place. There is only one Mt. Rushmore, one Eiffel Tower, and one Mona Lisa. Even copies are, themselves, distinct at some level. Two chairs of the same design are, nevertheless, two independent chairs. The destruction of one, for instance, does not immediately imply the destruction of the other.

We use this real-world attribute to conclude further facts about the world. For example, imagine that two people, A and B, each state separately that they are currently in an elevator, and, by the elevator's serial number, we know it is the same elevator. Furthermore, assume that both A and B state they are in the elevator at exactly the same time. The real-world fact list is now this:

$\mathrm{A}$ is in elevator $\mathrm{E}$ at time $\mathrm{T}$.

$B$ is in elevator $E$ at time $T$.

From this, you could conclude that both A and B are in the elevator together:

$A$ and $B$ are in elevator $E$ at time $T$.

It would be reasonable to further conclude that both $\mathrm{A}$ and B can see each other, perhaps talk to each other, and, if one pushed the elevator's stop button, the other would also be stopped.

This string of inferences about Person A, Person B, the elevator $E$, and time $T$ hinges on the assumption that there is only one A, one B, one $\mathrm{E}$, and one time $\mathrm{T}$. We could state this as a single existence law:

There is only one of anything. 
This is not necessarily true in VRML. To view a VRML file, the file's data are uploaded from the Web and into a local VRML browser application. Once displayed by the browser, the user can walk about in the virtual environment. Any number of users can upload the same VRML file to their own computers and display it at the same time. Each user gets a copy of the world and sees only it. Changes made to the world by one user do not necessarily affect all other users viewing their own independent copies of a VRML world. So, if Person A in an elevator $E$ in world copy $W 1$ pushes the elevator's stop button, Person B in the same elevator E in world copy W2 won't necessarily stop. The metaphor breaks down.

\section{Persistence}

In the real world, things do not just disappear spontaneously. If you put an object down, walk away, then return later, the object should still be there (in the absence of other forces to move it). Similarly, if you toss an object through an open door, then follow it into the next room, you should find the object there. Objects persist: They exist independent of our own existence. A chair, for instance, exists whether or not you are looking at it, or whether or not you are even in the same room with it. Using such a notion, the answer is simple to the philosophical puzzler "If a tree falls in the forest when no one is around, does it make a sound?" Since the tree, forest, and sound-transmitting atmosphere exist independent of a listener's existence, of course the tree will make a sound when it falls (though there are philosophers who would disagree).

Object persistence is at the core of our assumptions about the real world. However, persistence is not necessarily a feature of VRML virtual worlds. When users put an object down in a virtual room, they are editing that room's description, inserting the object into that room's VRML file. Since every user viewing a VRML environment has their own copy of that environment, changes they make are not propagated back to the original master Web copy of the environment. If the user leaves the room, the copy is deleted, along with the inserted object. Later, when the user returns, a fresh copy of the VRML environment is re-uploaded from the Web. The object inserted into the now-deleted local copy of the environment is not there. The object has not persisted in the absence of the user's direct attention. The metaphor has broken down.
Similar problems arise with actions performed within a VRML world. Imagine a room with an Earth globe. A user enters, reaches out, spins the globe, then leaves. In the real world, the globe would continue to spin for awhile. In VRML, it may not. The spinning of the globe must be controlled by a program that, repeatedly, turns the globe a little bit every few milliseconds. If the user is no longer in the room, the VRML browser may delete the globe-spinning program. Later, if the user returns, the globe will not be spinning. The globe's motion has not persisted in the absence of the user. Again, the metaphor has broken down.

\section{Metaphor Problems}

Metaphor breakdown problems such as those above are artifacts of the Web, rather than deficiencies of VRML. On the Web, users can author their own documents in any way they see fit. Two HTML documents linked together need not share the same formatting conventions. Similarly, two VRML documents linked together need not share the same user interface metaphor. Legislating a single user interface metaphor and patching all possibilities for metaphor breakdown is neither desirable nor practical. Metaphor breakdown is a necessary, though unfortunate, side effect of expressive freedom.

\section{CONCLUSIONS}

Technologies such as VRML enable users to author and hyperlink Web content in ever more complex ways. Using VRML, authors can create full 3-D virtual environments, weaving the Web into a vast architectural space of rooms, buildings, cities, and worlds. The many challenges we face include the design of appropriate user interface metaphors and adaptation when those metaphors break down. We can look forward to an exciting, if a bit chaotic, future.

\section{REFERENCES}

Ames, A., Nadeau, D., \& Moreland, J. (1996). The VRML sourcebook. New York: Wiley.

Bell, G., PARISI, A., \& Pesce, M. (1995). The virtual reality modeling language, Version 1.0 [On-line]. Available: http://www.wired.com/ vrml.tech/vrml10-3.html

RAGGETT, D. (1995). Hypertext markup language specification, Version 3.0 [Internet draft]. Available: http://www.w3.org/hypertext/ www/markup $/ \mathrm{htm} 13 /$ coverpage.html

(Manuscript received November 13, 1995 revision accepted for publication February 16, 1996.) 